\title{
An Exponential Melanoma Trend Model
}

\author{
Örjan Hallberg*
}

\author{
Hallberg Independent Research, Brattforsgatan 3, 12350 Farsta, Sweden
}

\begin{abstract}
The present study investigated whether whole population exposure to radiation introduced by radio broadcasting and cell phone systems might explain recent increases in melanoma trends in Nordic countries or not. Trends were modeled using a single exponential function of the time each age group has been living in the new environment since an environmental change took place. The results clearly show that melanoma incidences started to increase exponentially by the time lived as an adult since 1955 and that a second trend break occurred in 1997. We searched best fit between calculated and reported age-standardized rates by parameter variation, and compared calculated with reported age-specific rates without further parameter adjustments. Local variations of breast cancer, lung cancer and all cancers together significantly correlated with corresponding local melanoma rates in Sweden. Increasing cancer trends since around 1997 seem related to a population covering environmental change effective from early 90's. We conclude that this exponential trend model can be a useful tool in understanding responses to sudden environmental changes.
\end{abstract}

Keywords: Cancer, Melanoma, Cell phone, Speech time, Incidence, Trends.

\section{INTRODUCTION}

In January 2014, the World Health Organization (WHO) issued a press release stating that the cancer burden during the next 20 years will increase from 14 million new cases per year to 25 million. In the Nordic countries we have noticed a considerable increase in cancer rates over the last 20 years. The first time that total cancer rates suddenly started to increase was in 1955. The change was quite abrupt the first years, and the rate increased from 232 cases per 100000 persons in 1955 to 488 cases per 100000 persons in 1996, when it seemed to stabilize in Sweden. However, from 1997 the cancer rate started to increase again, reaching 602 cases per 100000 inhabitants in 2012.

In Sweden, the number of new melanoma cases per year has increased 2.5-fold from 1337 in 1992 to 3368 in 2012. Figure $\mathbf{1}$ shows more specifically the development of annual new melanoma cases in the Nordic countries together.

Melanoma incidence and mortality have been increasing steadily since mid $20^{\text {th }}$ century. Traditionally, increased sun tanning habits have been blamed as the main cause of this public health problem. However, both mortalities and incidences have stabilized among younger age groups up until year 2000, while continued to increase in an exponential way among the older groups. This fact suggests that the melanoma risk suddenly increased for the whole population and that those younger age groups, once having lived all their lives in this new environment, then now should be

*Address correspondence to this author at the Hallberg Independent Research, Brattforsgatan 3, 12350 Farsta, Sweden; Tel: +46-8-605 4998;

E-mail: oerjan.hallberg@swipnet.se expected to show stabilizing rates. In 2008 the author presented a trend model for melanoma incidences, [1] where an environmental change was assumed capable to reduce cell repair efficiency from a specific point in time. A less complex exponential model for melanoma mortality was presented more recently [2]. Even though a simple exponential function may look superficial, its simplicity may also become a strength in case it really is capable of modeling varying cancer trends using few model parameters.

The first increase after 1955 has been linked to the introduction of FM radio broadcasting, which forced the whole population to sleep every night with skin currents driven by the body-resonant fields created by the main radio transmitters. From October 1997 the health of the general population of Sweden suddenly degraded, with increasing numbers of people staying home from work on sick-leave; see Figure 1 and ref. [3]. The sudden increase from that time included both short and long term sickness. This health problem was most predominant in areas where cell phones used high output power to connect with the nearest base station, i.e. the less populated regions of Sweden. We also know that from 1997 the use of cell phones in the new $1.8 \mathrm{GHz}$ band boomed, Figure 1 .

The objective of the present study was to analyze available population statistics to determine if the increasing melanoma rates after 1997 can be explained as a response to the massive exposure of the whole population to base station and cell phone radiation since that time.

If the suddenly enforced microwaving of the whole population from 1997 can cause increased melanoma 


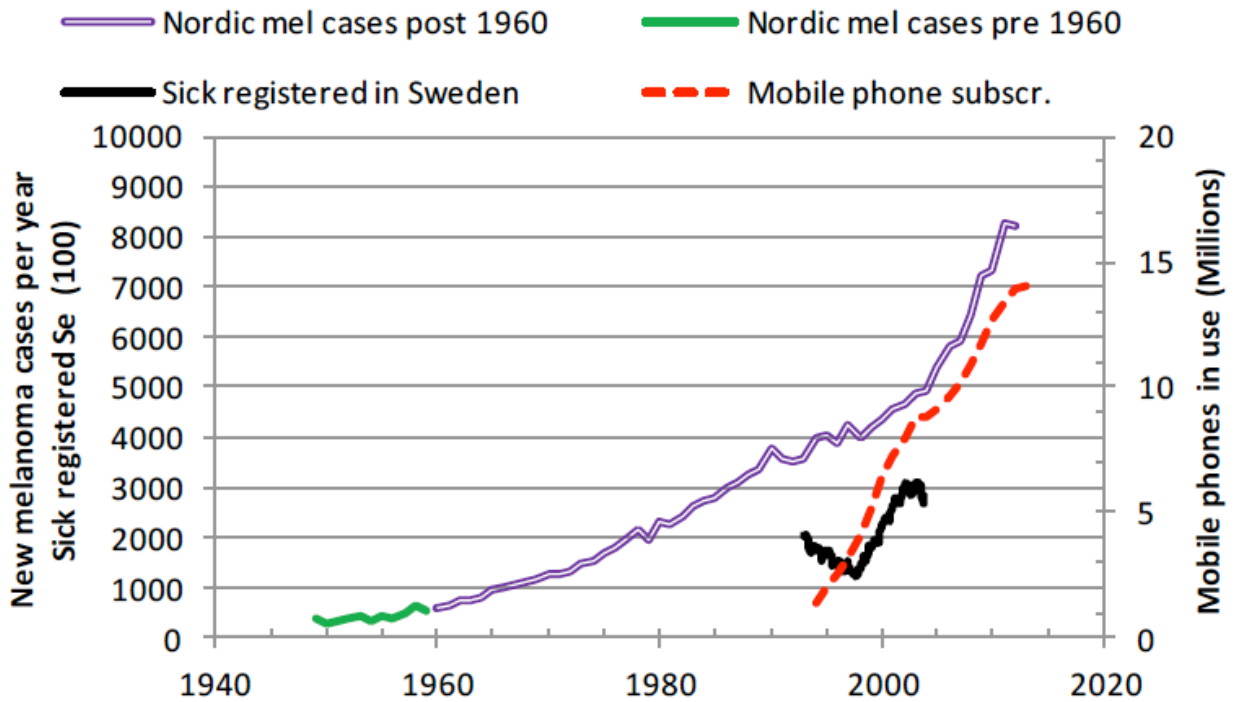

Figure 1: Annual numbers of melanoma cases in the Nordic countries. Also shown are the number of cell phones in use over time in Sweden, and number of sick registered in Sweden from 1993 to 2003.

rates vs. exposure time, the exponential model would be as useful after 1997 as it has been to model trends after 1955. If there is a biological connection between high frequency radiation and cancer development, it would also be possible to estimate future cancer rates for different scenarios of cell phone use and an estimation of the effect from fictive changes made in earlier years. Thus, the main aims of this study were to collect relevant data, develop a simple application for melanoma trends analysis to project different scenarios of interest.

\section{METHODS}

Cancer data from 1958 and later were retrieved from the Swedish National Board of Health and Welfare and also from the NORDCAN data base for all Nordic countries. For the time period 1912 to 1957, we used library information available at Statistics Sweden. Melanoma rates were only available from 1958. The basic model for cancer trends was similar to the one earlier described in ref. [2] used for melanoma mortality. If cell damages start adding up every year from a specific point in time, then the cancer risk will follow a "growth -by-growth" function in case cell repair efficiency does not keep pace with the new damages. Such a development may be modeled by an exponential function of time lived in the new environment.

Since the initial exponential increase is assumed to be similar for all age groups, it is easy to create a simple exponential model that applies to all ages using the following key parameters:
- $\quad \mathrm{A}=$ age group, e.g. $70-79$ years old

- $\quad \mathrm{Y}_{0}=$ the year in which a sudden environmental change occurred

- $\quad \mathrm{Y}=$ calendar year

- $\quad T=Y-Y_{0}$; the number of years since the environmental change; $Y>Y_{0}$

- $\quad P=$ age from when the environmental change has any effect, e.g. 15 years.

- $\quad A_{0}=$ Incidence in year $Y_{0}$ for age group $A$

- $\quad \mathrm{a}=$ constant multiplied by the exponential function

- $\quad b=$ the slope used in the exponential function for incidence

This can be described as follows. The incidence of age group $A$ is assumed to be at a constant level $A_{0}$ before year $Y_{0}$. After year $Y_{0}$ the incidence will increase until all people of age $A$ have been living all their lives since age $P$ in the new environment. When that happens, the incidence for age group $A$ is assumed to stabilize at a level $\mathrm{I}(\mathrm{T}, \mathrm{A})$ as given by equation (1).

$I(T, A)=A o+a *(\exp (b *(A-P))-1)$

In order to describe the incidence trends after year $Y_{0}$, during the exponentially increasing phase and finally after the stabilization, we formatted the total equation as an ordinary Excel IF-expression given by equation (2). 


$$
\begin{aligned}
& I(T, A)=I F(T<A-P ; A o+a *(\exp (b T)-1) \\
& A o+a *(\exp (b *(A-P))-1))
\end{aligned}
$$

We used equation (2) to calculate the agestandardized rates $\left(\mathrm{ASR}_{\mathrm{w}}\right)$ of melanoma incidence. In searching for best fit between calculated and reported rates, we used the Problem Solver unit within Excel and varied the constant (a) and the exponential slope factor $(\boldsymbol{b})$. We also varied the starting age, $\boldsymbol{P}$, to see if there would be any difference between 1955 and 1997 for best fit. In order to test the validity of this approach we then compared calculated age-specific incidences with reported data without any further parameter variations.

Because the trend break in 1997 was the second one after 1955 (Figure 1), we had to calculate the increase above the cancer rates projected based on reported data from 1955 to 1996 in case no further sudden trend change had occurred. This basic, undisturbed trend was calculated using the exponential function of incidence vs. life time spent in the new environment since 1955, as earlier described. Thus the total cancer incidence was the original 'non-disturbed' trend from 1955 plus the additional increase emanating from the second environmental change from 1997, using $a_{2}{ }^{*}\left(\exp \left(b_{2} T\right)-1\right)$ as the added function where $T$ is the time since 1997.

In order to estimate the effect from a reduced or completely stopped exposure from the radiation sources involved, we did as follows: If the exposure is stopped in year $Y_{1}$, then the incidence at time $Y=Y_{1}+t$ for age group $A$ was estimated according to equation (3).

$$
I(T, A)=(A-t) o+a *(\exp (b(T-t))-1)
$$

Where (A-t) is the initial incidence before e.g. 1955 or 1997 for the age group A-t.

Our model covers a cancer incidence trend influenced by two environmental changes active from 1955 and 1997 respectively. The application makes it easy to simulate trend responses in case one or both environmental factors ceased to exist from a specific year.

\section{RESULTS}

The development of the age-standardized incidence of skin melanoma in Sweden is shown in Figure 2. Here the calculated projection is based on the exponential model, equation (2). Since the first use of mobile phones (cell phones) started in 1981, the annual usage of such devices has increased very fast, especially after 1997, Figure 2. After parameter variation, the best fit was determined, see Table 1.

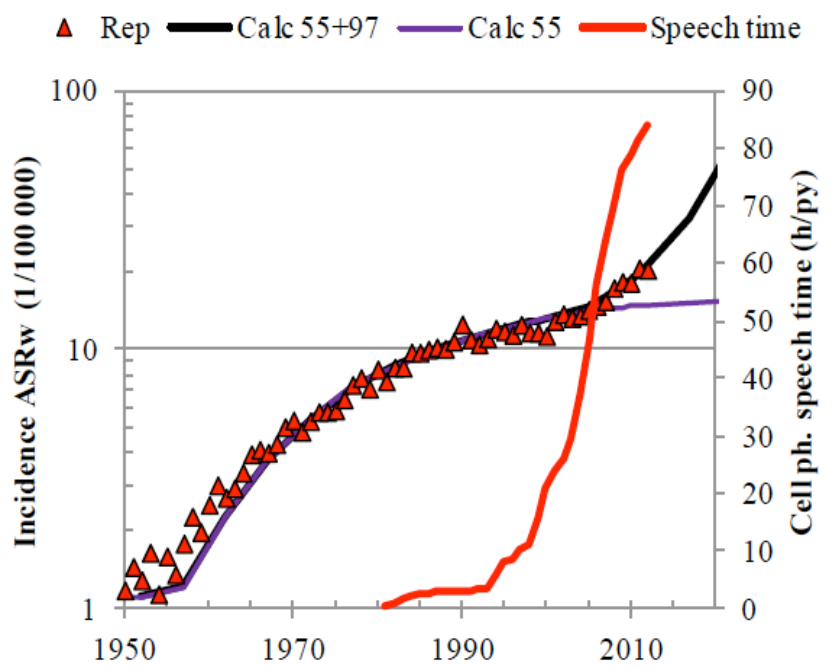

Figure 2: Reported and calculated age-standardized rates of skin melanoma in Sweden. Also shown is the calculated trend based on the environmental change in 1955 only, and the average speech time per person-year in Sweden. Data from 1950 to 1957 represent Denmark in lack of Swedish data.

Table 1: Parameters for the Two Exponential Functions Used in the Trend Models

\begin{tabular}{|c|c|c|c|}
\hline Cancer form & $\begin{array}{c}\text { Constant } \boldsymbol{a} \\
\boldsymbol{a}^{*} \exp (\boldsymbol{b} \boldsymbol{T})\end{array}$ & $\begin{array}{c}\text { Exponent } \boldsymbol{b} \\
\boldsymbol{a}^{*} \exp (\boldsymbol{b} \boldsymbol{T})\end{array}$ & $\begin{array}{c}\text { Start age } \boldsymbol{P} \\
\text { (Years) }\end{array}$ \\
\hline \hline Melanoma 1955 & 10.355 & 0.0362 & 15 \\
Melanoma 1997 & 0.34 & 0.2211 & 5 \\
\hline
\end{tabular}

To test the quality of the model, we plotted the calculated age-specific incidences according to equation (2) without any further parameter variations, Figure 3. It should be noted that calculated data in Figure $\mathbf{3}$ are results from the parameter fitting done in Figure 2. Thus, the fact that also age-specific data in Figure $\mathbf{3}$ fit well with reported data is in support of our simple model approach.

The corresponding parameters for the agestandardized melanoma trend model are listed in Table 1.

In case the use of cell phones continues to increase, as it has since 1994, the calculated incidence in 2020 would be 46 per 100000 py, Figure 2. If both cell phone use and FM broadcasting were to be stopped in 2017, the incidence in 2022 would be 25.8 as shown in Figure 4 using also Equation (3). 


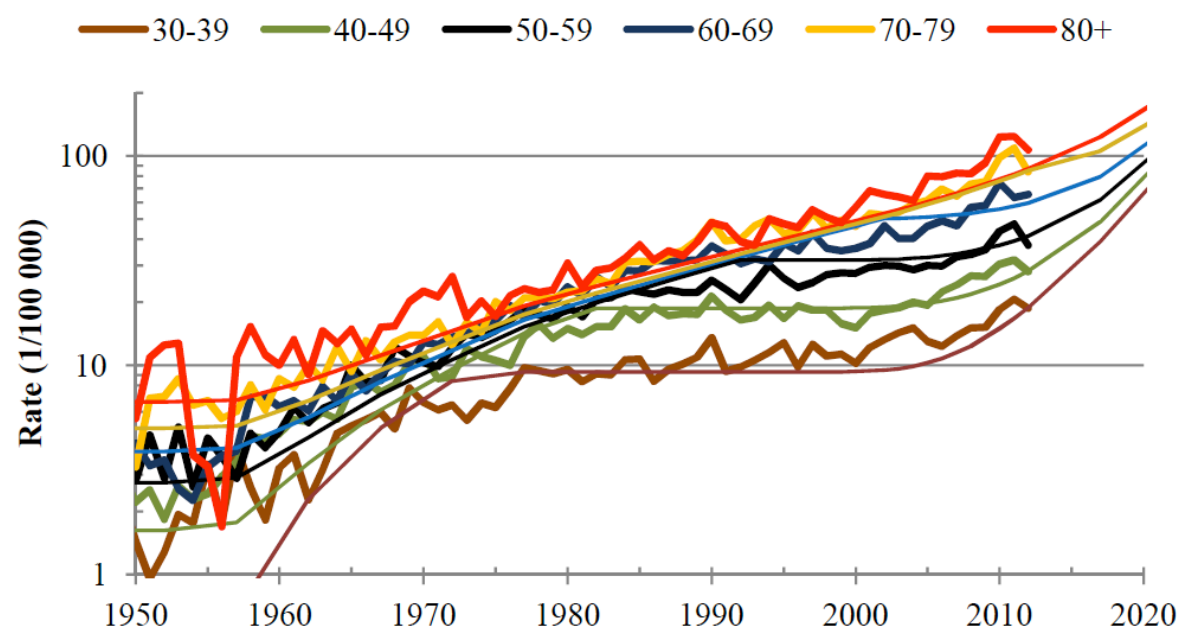

Figure 3: Age-specific incidence of melanoma in Sweden as reported and calculated.

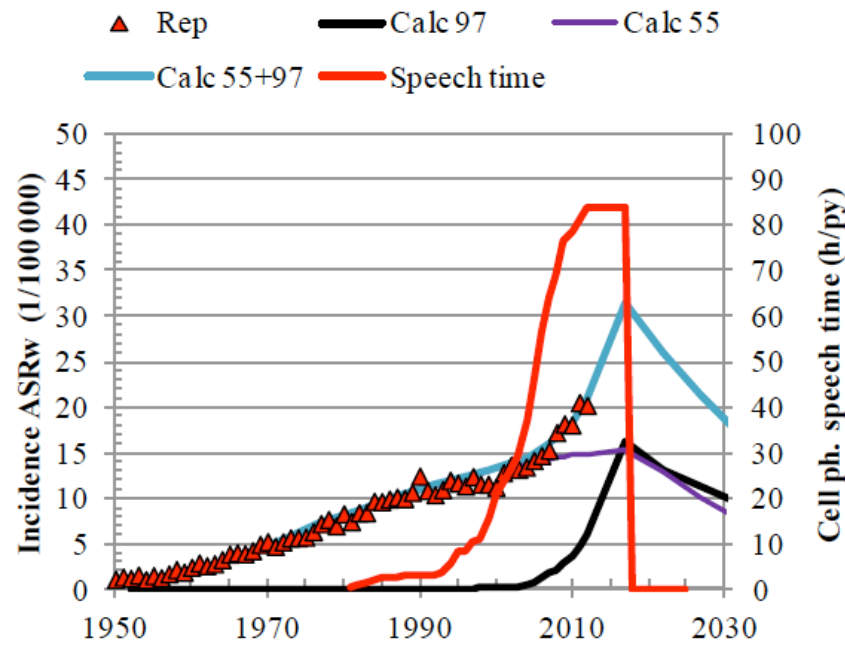

Figure 4: The graph shows the increasing rates of melanoma in Sweden. The calculated solid line shows the response to a ceased use of cell phones and FM broadcasting after 2017.

In order to test the hypothesis of UV-generated melanoma cases we performed a multi-regression analysis of incidence vs. FM transmitter coverage and UV radiation power density. The analysis gave a significant association to transmitter density $(p=1.2 \mathrm{E}$ 4) and a non-significant association to UV radiation ( $p$ $=0.45)$. See Table 2 for regression analysis of melanoma, lung cancer, breast cancer and all cancers together against transmitters, UV radiation and smoking prevalence in the 21 Swedish counties.

\section{DISCUSSION}

Figure 1 gives strong indication of sudden environmental changes in Sweden from 1955, as well as from 1997. The increasing number of sick people registered in Sweden since October 1997 should have alerted the responsible authorities to search for an explanation and to enforce corrective actions.

With the post-1955 increase, the annual increase of melanoma and also all cancer cases together started to

Table 2: Multi-Regression Analysis of Different Cancers Performed Against Environmental Factors and Corresponding p-Values. Results in Bold Numbers are Significant.

\begin{tabular}{|c|c|c|c|c|}
\hline Cancer type, years & FM Transmitters & UV radiation density & Population density & Smoking prevalence \\
\hline \hline Melanoma 1986-93 & $\mathbf{0 . 0 0 3 8}$ & 0.0926 & & \\
\hline Melanoma 2004-12 & $\mathbf{0 . 0 0 0 1 1 6}$ & 0.4508 & & \\
\hline Breast can. 1986-93 & $\mathbf{0 . 0 2 9 6}$ & 0.286 & 0.334 & 0.702 \\
\hline Breast can. 2004-12 & 0.649 & 0.336 & & 0.198 \\
\hline Lung can. 1986-93 M & $\mathbf{0 . 0 1 6 7}$ & & 0.061 \\
Lung can. 1986-93 W & 0.076 & & & 0.0462 \\
\hline Lung can. 2004-12 M & $\mathbf{0 . 0 0 1 2}$ & & $\mathbf{0 . 0 0 0 6}$ & 0.218 \\
\hline Lung can. 2004-12 W & $\mathbf{0 . 0 3 4 3}$ & & & 0.431 \\
\hline All cancers 1986-93 & 0.352 & & & \\
\hline All cancers 2004-12 & 0.723 & & & 0.121 \\
\hline
\end{tabular}


diminish to approach stable incidences after 1998. But in 1997 a sudden increase was seen the first year, and during the following years the annual increases tended instead to accelerate even more, indicating an exponential problem. The stabilizing trend before 1997 may be explained by the increased cancer rate after 1955 being due mainly to reduced cell repair capacity. By 1998 the main part of the population had been living in this new environment all of their lives, so stabilization at the new level would seem logical. After 1997, however, the annual increase accelerated, possibly due to a combination of increased original cell damages and further reduced cell repair capacity. Thus, an environmental change with capacity to create cell damage should be sought. It is interesting to note that the incidence increase since 1997 above the basic incidence calculated from 1955 is the same for all age groups older than 30 years, suggesting $24 / 7$ base station radiation exposure as the main cause rather than cell phone use time, which varies between age groups.

In 2014 a theory about the influence from electromagnetic radiation on T-lymphocytes was published by Vincent Lauer, [4]. According to this theory body-resonant radiation was expected to affect skin melanoma more drastically than other cancers due to the "skin effect" (high frequency currents tend to concentrate to the periphery of a conductor) and body penetration. From a quantum physics perspective higher frequency radiation up to $3 \mathrm{GHz}$ was also expected to enhance cancer development, which is in line with the drastically increasing melanoma rates noticed in Nordic countries since 1997.
If the hypothesis is right about the cancer promoting effect from body-resonant radiation, we should expect to find higher incidences in regions covered by several body resonant transmitters than in regions covered by only one or (as before 1955) by none. To test the hypothesis we calculated reported cancer incidences relative to rates reported in late 50's for all the 21 counties in Sweden. Since each county also can be characterized by an average number of covering FM transmitters over the population, it was easy to plot reported cancer incidences relative to rates in 1958 vs. average number of covering FM transmitters. Already in 2002 an extremely strong association was found in Sweden between the number of covering FM main transmitters and melanoma incidence in the 289 different municipalities (communes) in Sweden. The correlation was characterized by $p=3 \mathrm{E}-23$ which hardly can be explained as being just a matter of coincidence. It also turns out that the average body height correlates significantly with the number of surrounding FM transmitters, [5]. Thus body-resonant radiation appears to affect both growth hormones (IGF1 ) and cancer preventing hormones like melatonin etc.

In a pooled cohort study Wirén et al. [6] found a significant correlation between cancer incidence and body height. In that study the strongest correlation to body height was found for melanoma, both for men and women.

Figure 5 gives the results for melanoma, lung cancer, breast cancer and all cancers together. Obviously there are strong correlations between cancer rates and the number of body-resonant transmitters

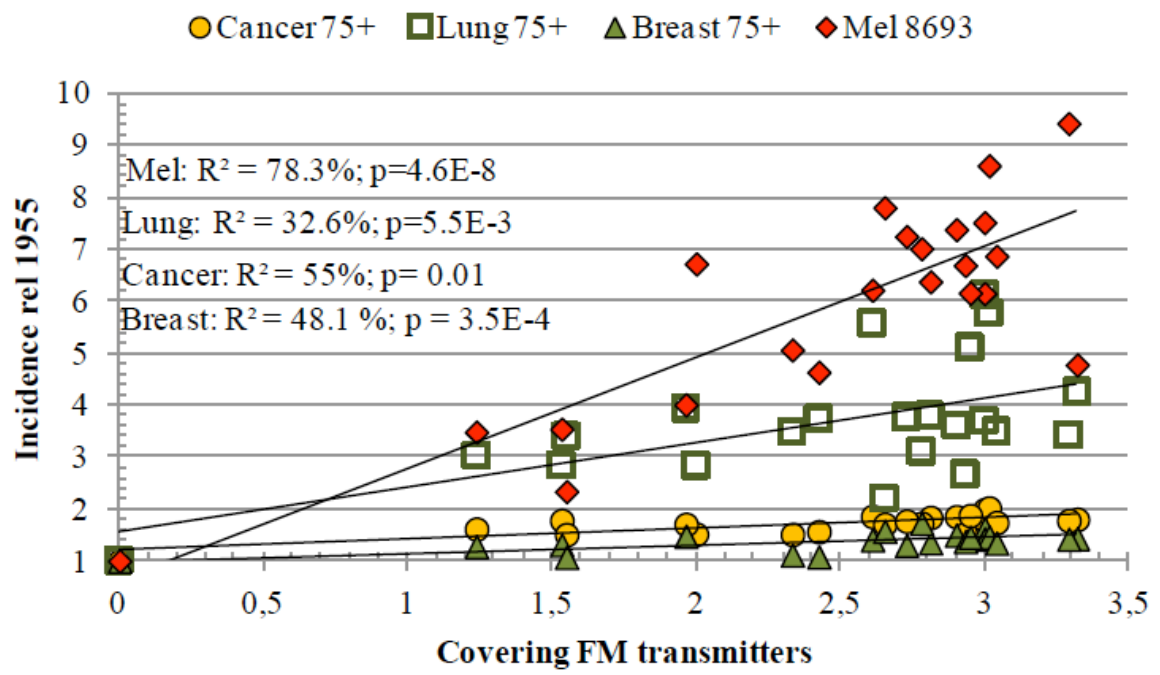

Figure 5: Cancer incidence relative to rates reported in 1958 vs. average number of covering FM transmitters in the 21 different counties of Sweden. Incidence rates were average data collected during the time period 1986-1993 for elderly (75+) and for all ages ASRw (melanoma). 
Lung $93 \times$ Lung $12 *$ Breast $93 \bigcirc$ Breast $12 \diamond$ Cancer $93 \Delta$ Cancer 12

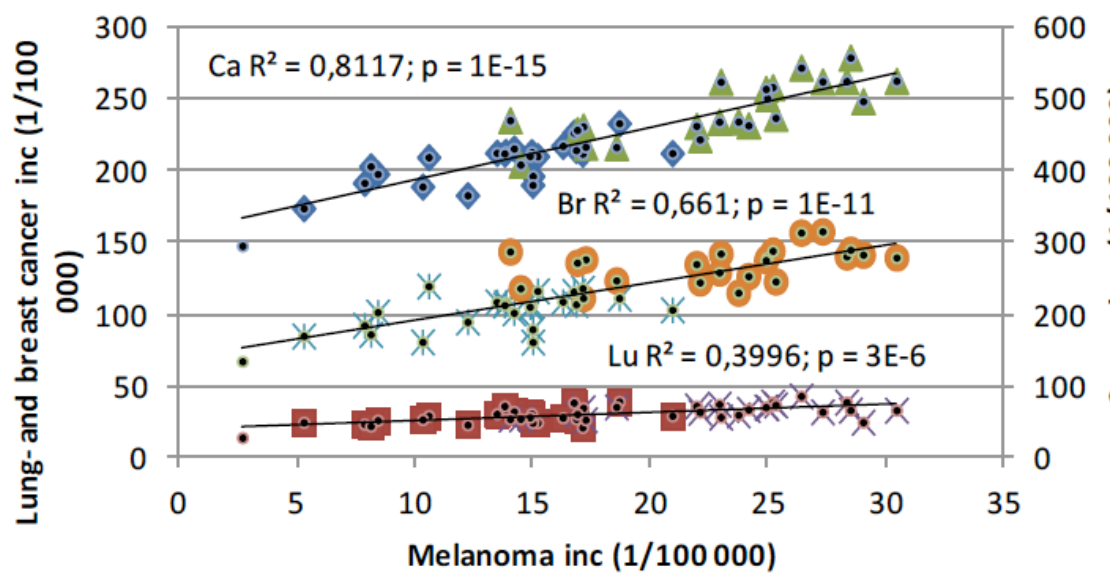

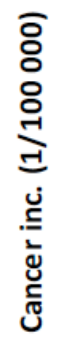

Figure 6: Lung cancer, breast cancer and total cancer incidences plotted vs. melanoma incidence in the 21 Swedish counties during the time periods 1986-1993 and 2004-2012. The first points at Mel inc $=2.6$ represent readings in 1958 when FM transmitters had been in use for only three years.

covering the 21 Swedish counties. As assumed in the theory paper by Lauer [4], melanoma has the most significant association to the number of surrounding FM transmitters.

It also turns out that cancer, lung cancer and breast cancer are stronger associated with melanoma incidence than with transmitters. Thus melanoma incidence might be an even better indicator of the local radiation environment and its exposure duration than simple counts of overlapping circles from radio coverage maps. In order to test this assumption we plotted lung cancer, breast cancer and total cancer average incidences vs. reported melanoma incidences in our 21 counties during the time periods $1986-1993$ and $2004-2012$, see Figure 6.
The apparently very strong associations between melanoma incidence and other cancers in the 21 different counties of Sweden, speak against the common believe, that more sun screens should be the final solution to the problem of increasing melanoma rates.

Figure 7 shows melanoma incidence over time for counties covered by $<2$ transmitters and counties covered by more transmitters.

\section{CONCLUSIONS}

The exponential trend model presented here is capable to explain both age-standardized and age specific trends using very few parameters compared

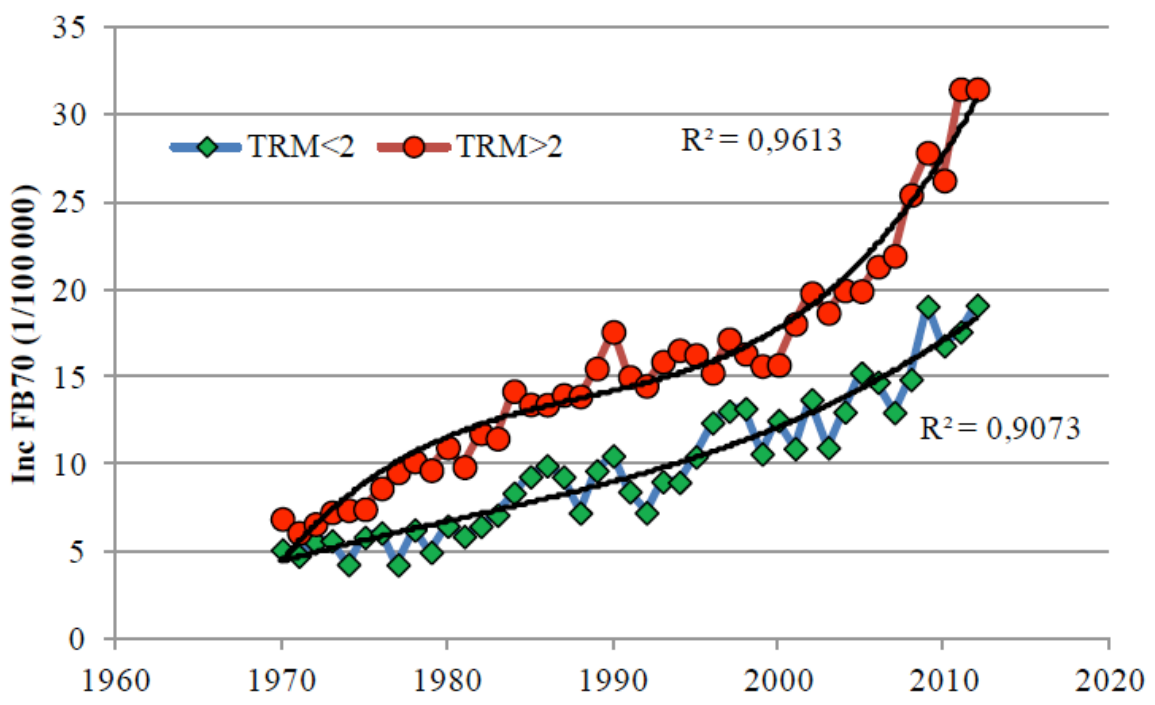

Figure 7: Melanoma incidence in Swedish counties covered by $<2 \mathrm{FM}$ transmitters is significantly less than in counties covered by more transmitters over calendar time. 
with what traditional Age-Period-Cohort (APC) models normally require. Since the model also is based on physical facts, like the starting year for an environmental change and the age from which it may have an effect on cancer risks, it should be used in cancer trend projections.

The main conclusion from this model study is that there is a risk that we will encounter fast and expensive increases in the incidences of several types of cancers. Further detailed studies on causative factors behind these alarming trends are needed. If the model projections are correct, we have to face that the population today has already accumulated cell damage that will develop into cancer in the next 10 years. The study indicates, however, that there is a possibility that increasing cancer rates can be reverted and that hospital costs and lives can be saved if relevant actions are taken.

Responsible authorities need to consider what to do. The cell phone industry needs to concentrate immediately on the next generation of mobile communications technology e.g. based on the IR/UV radiation we have adapted to living with safely for millions of years.

\section{FUNDING}

This work was sponsored by internal funds within Hallberg Independent Research only. Hallberg Independent Research was founded in 2001 and is registered by the Swedish Patent and Registration Office.

\section{REFERENCES}

[1] Hallberg Ö. A reduced repair efficiency can explain increasing melanoma rates. Eur J Cancer Prevent 2008; 17: 147-152.

http://dx.doi.org/10.1097/CEJ.0b013e3282b6fceb

[2] Hallberg Ö. An Exponential Model for Melanoma Mortality Trends. Int J Statist Med Res 2013; 2: 200-03. http://www.lifescienceglobal.com/home/cart?view=product\&id $=692$

[3] Hallberg Ö, Johansson O. Apparent decreases in public health indicators after 1997 -Are they due to improved diagnostics or to environmental factors? Pathophysiology 2009; 16: 43-46.

http://dx.doi.org/10.1016/i.pathophys.2008.12.004

[4] Lauer V. A model of the interaction of $T$ lymphocytes with electromagnetic waves. Hyper Articles en Ligne 2014; HAL: hal-00975963, version 1; http://hal.archives-ouvertes.fr/hal00975963

[5] Hallberg Ö. Cancer and body height. Pathophysiology 2014; 21: 177-181. http://dx.doi.org/10.1016/i.pathophys.2014.05.001

[6] Wirén S, Häggström C, Ulmer $\mathrm{H}$, Manjer J, Bjørge T, Nagel $\mathrm{G}$, et al. Pooled cohort study on height and risk of cancer and cancer death. Cancer Causes Control 2014; 25: 151-159. http://dx.doi.org/10.1007/s10552-013-0317-7

(c) 2015 Örjan Hallberg; Licensee Lifescience Global.

This is an open access article licensed under the terms of the Creative Commons Attribution Non-Commercial License (http://creativecommons.org/licenses/by-nc/3.0/) which permits unrestricted, non-commercial use, distribution and reproduction in any medium, provided the work is properly cited. 